\title{
Efecto de la Suplementación con Medicago sativa (Alfalfa) sobre Algunos Parámetros de la Leche Bovina
}

\author{
Effect of Supplementing Medicago sativa (Alfalfa) on Some Parameters of \\ Bovine MiLK
}

\author{
Anastasia Cruz Carrillo ${ }^{1}$, Carlos Sebastián Lizarazo Cely ${ }^{1}$, José Luis Porras Vargas ${ }^{1}$
}

\section{Resumen}

El objetivo de este trabajo fue determinar si la suplementación con alfalfa en vacas en producción podría generar un efecto antioxidante y modificar la composición de los ácidos grasos de la leche. Se utilizaron 19 vacas distribuidas en un grupo tratado (suplementado con alfalfa; $n=11)$ y un grupo control $(n=8)$. Se determinó el nivel de oxidación de la grasa de la leche (prueba de jabón de cobre), la calidad composicional y la presencia de ácidos grasos (cromatografía de gases) en la leche. La grasa de la leche de los animales suplementados mostró mayor estabilidad y menor oxidación respecto al grupo control, mostrando un efecto antioxidante, aun en aquella leche que se mantuvo 14 días a temperatura ambiente. Asimismo, hubo aumento de ácidos grasos poliinsaturados y disminución de los saturados, y aumento del porcentaje de grasa total de la leche. Se concluye que la suplementación con alfalfa da mayor estabilidad a la grasa de la leche por su efecto antioxidante y mejora la proporción de ácidos grasos poliinsaturados con disminución de los saturados, lo cual podría ser más benéfico para la salud del consumidor.

Palabras clave: grasa; oxidación; lucerna; flavonoides

\section{Abstract}

The aim of this study was to determine whether alfalfa supplementation in milking cows could generate an antioxidant effect and modify the composition of milk fatty acids. Nineteen cows were distributed in a treated group (supplemented with alfalfa; $n=11$ ) and a control group $(n=8)$. The level of oxidation of milk fat (copper soap test), compositional quality and the presence of fatty acids (gas chromatography) in milk were determined. The

\footnotetext{
${ }^{1}$ Grupo GIBNA, Programa de Medicina Veterinaria y Zootecnia, Universidad Pedagógica y Tecnológica de Colombia, Tunja, Colombia

${ }^{2}$ E-mail: anastasia.cruz@uptc.edu.co
} 
milk fat of supplemented animals showed greater stability and less oxidation compared to the control group, showing an antioxidant effect even in milk kept during 14 days at room temperature. There was an increase of polyunsaturated fatty acids and a decrease of the saturated ones, and the percentage of total milk fat increased. It is concluded that alfalfa supplementation gives greater stability to milk fat due to its antioxidant effect, without affecting the reproductive performance of cows and improves the proportion of polyunsaturated fatty acids with a decrease of saturated ones, which would be more beneficial to consumer health.

Key words: fat; oxidation; lucerne; flavonoids

\section{INTRODUCCIÓN}

Medicago sativa (lucerna o alfalfa), es una leguminosa perenne de la familia Febaceae (Benzada, 2010), ampliamente utilizada en nutrición animal como fuente de proteína. Las nuevas tendencias que buscan no solo alimentar sino aportar compuestos para atender la salud humana y animal, así como producir alimentos de origen animal enriquecidos, han resaltado el uso de alfalfa por la presencia de metabolitos secundarios, como los flavonoides (Gohlke et al., 2013). Estos compuestos polifenólicos, con una estructura común a todos, el glucósido benzo$\gamma$-pirano (Cartaya y Reynaldo, 2001), son reconocidos como metabolitos antioxidantes y captadores de radicales libres (Rana et al., 2010).

Los flavonoides de algunas leguminosas, incluyendo la alfalfa, también tienen efecto xenoestrogénico, utilizado con intenciones terapéuticas, pero también identificados como inductores de infertilidad en animales (Benzada, 2010; Al-Dosari, 2012). Las principales isoflavonas son genisteína daidzeina y glicetina (Trinácty et al., 2009) unidas a carbohidratos, que se encuentran en la alfalfa y otras leguminosas (Woclawek-Potocka et al., 2013). Una vez que las isoflavonas son consumidas por los rumiantes, las bacterias ruminales hidrolizan el enlace carboxílico dejándolas como agliconas; luego la daidzeina es convertida a equol, el cual tiene una capacidad estrogénica alta, y la genisteína a p- etil-fenol. El rumen requiere cerca de 10 días para adaptarse a esos cambios y estabilizar el metabolismo de las isoflavonas. Las agliconas son absorbidas en el rumen y biotransformadas por glucuronidación y sulfatiación en el hígado, para luego pasar a circulación general, donde pueden encontrarse en altas concentraciones en la sangre (Woclawek-Potocka et al, 2005; Piotrowoska et al., 2006), aunque su tiempo de vida media es corto. Finalmente, los compuestos son eliminados en forma activa por la orina y la leche (Setchell et al., 2003; Trinácty et al., 2009).

Con base en diferentes estudios, donde destacan los de Antignac et al. (2004), Hoikkala et al. (2007) y Steinshamn et al. (2008), se afirma que las isoflavonas y sus metabolitos (principalemente el equol), se encuentran en altas concentraciones en la leche de vacas suplementadas con leguminosas como tréboles, soya, alfalfa. Trinácty et al. (2009) y Kilic y Lindsay (2005) reportan altas concentraciones de daidzeina y equol en leche de vacas y cabras suplementadas con soya. Con lo anterior, se presume que la presencia de isoflavonas y sus metabolitos activos en la leche pueden constituir una fuente de antioxidantes para las personas y que la inclusión de flavonoides en la dieta sería el factor determinante de su presencia en la leche (De Feo et al., 2006).

El objetivo del trabajo fue evaluar la acción antioxidante de Medicago sativa en la leche de vacas suplementadas con alfalfa, 
evaluando la calidad composicional de la leche, el proceso de rancidez y la presencia de ácidos grasos en la grasa.

\section{Materiales y Métodos}

\section{Lugar y Animales de Estudio}

Los animales del estudio eran parte de la Granja Experimental Tungüavita de la Universidad Pedagógica y Tecnológica de Colombia (UPTC), ubicada en el municipio de Paipa, Boyacá (Colombia). Paipa está ubicada en la cordillera oriental colombiana, a $2525 \mathrm{msnm}$, con $13{ }^{\circ} \mathrm{C}$ de temperatura promedio y $738 \mathrm{~mm}$ de precipitación pluvial anual.

Se seleccionaron 22 hembras en el primer tercio de lactancia, aparentemente sanas, con antecedentes de normalidad reproductiva y que hubieran tenido por lo menos una cría. Los animales incluidos fueron de las razas Holstein, Simmental, Jersey y cruces de estas razas; se encontraban vacunados contra aftosa y brucelosis y estaban desparasitados. Se dividieron al azar en un grupo tratado y uno control, cada uno con 11 animales; no obstante, la granja vendió tres vacas del grupo control durante el estudio.

Los animales se mantuvieron al pastoreo sobre pasto kikuyo (Pennisetum clandestinum), en un sistema de rotación de potreros y con dos ordeños al día, donde recibían suplemento concentrado según sus niveles productivos. El manejo, lugar de mantenimiento y condiciones generales fueron iguales para todas. Las hembras suplementadas recibieron harina de alfalfa comercial durante cada ordeño, en cantidad equivalente a su peso vivo y producción de leche, siendo entre 3 y $4 \mathrm{~kg}$ por animal. La harina de alfalfa se suministró mezclada con el concentrado durante cuatro meses, además de un mes como periodo de acostumbramiento.

\section{Valoraciones y Procedimientos}

Las variables dependientes fueron oxidación de la grasa de la leche usando la prueba de jabón de cobre, composición de la leche y cuantificación de ácidos grasos.

Al cumplirse los cuatro meses de suplementación, se tomaron tres muestras de leche por animal. La primera $(10 \mathrm{ml})$ se envió refrigerada al laboratorio Mol LabsQuimiométricas para la cuantificación de ácidos grasos (AG) mediante cromatografía de gases, usando como blanco BHT una columna Rtx- $233060 \mathrm{~m}, 0.32 \mathrm{~mm}$ ID, $0.2 \mu \mathrm{m}$, el programa $100^{\circ} \mathrm{C} / 2 \mathrm{~min}, 250^{\circ} \mathrm{C} / 11 \mathrm{~min}$ $\left(21^{\circ} \mathrm{C} / \mathrm{min}\right)$, Split inicial: 25 , Split $3 \mathrm{~min}: 2$ y volumen de inyección $1 \mu \mathrm{l}$. El equipo usado fue un cromatógrafo de gases Varian 3900 , con detector de ionización de llama. La segunda muestra $(10 \mathrm{ml})$ se envió refrigerada al Laboratorio de Nutrición Animal, UPTC, para evaluar la calidad composicional (Lactoscam), valoración realizada el mismo día que se tomó la muestra, la cual se colectó después del ordeño de cada animal y posterior a su homogenización. La tercera muestra $(9 \mathrm{ml})$ fue dividida en tres viales y se utilizó para la determinación del efecto antioxidante de los flavonoides en la leche, mediante la cuantificación de ácidos grasos libres, utilizando la prueba de solvente de jabón de cobre modificada (Shipe et al., 1980) en el Laboratorio de Nutrición Animal, UPTC.

Para esta última prueba se utilizó el método de extracción de jabón de cobre modificado para medir ácidos grasos de la leche, según Shipe et al. (1980). La técnica se basa en la medición del ácido palmítico libre mediante espectrofotometría, cuya concentración es directamente proporcional al grado de oxidación de la grasa de la leche. La primera muestra se analizó en el momento que llegó al laboratorio, en tanto la segunda y tercera muestra se almacenaron a temperatura ambiente para analizarlas a los 7 y 14 días respectivamente, buscando determinar 
Cuadro 1. Concentración de ácido palmítico (ng/ml) en leche de vacas suplementadas durante cuatro meses con harina de alfalfa y en vacas control, donde la leche fue almacenada por 0,7 y 14 días a temperatura ambiente

\begin{tabular}{cccccccc}
\hline & \multicolumn{3}{c}{ Grupo tratado } & \multicolumn{5}{c}{ Grupo control } \\
\hline Vaca & Día 0 & Día 7 & Día 14 & Vaca & Día 0 & Día 7 & Día 14 \\
\hline 1 & 0.01 & 1.84 & 20.20 & 1 & 13.00 & 71.73 & 111.7 \\
2 & 0.03 & 3.64 & 23.80 & 2 & 2.74 & 27.40 & 55.96 \\
3 & 0 & 0 & 0 & 3 & 0 & 0.36 & 36.40 \\
4 & 22.00 & 41.05 & 60.20 & 4 & 9.40 & 47.38 & 63.92 \\
5 & 0.05 & 16.50 & 73.76 & 5 & 0.03 & 16.60 & 56.07 \\
6 & 0 & 0.09 & 1.66 & 6 & 43.15 & 63.46 & 78.07 \\
7 & 1.66 & 2.02 & 2.14 & 7 & 2.27 & 50.96 & 94.26 \\
8 & 0 & 0.31 & 25.60 & 8 & 7.60 & 40.65 & 74.50 \\
9 & 2.20 & 14.80 & 45.30 & & & & \\
10 & 40.00 & 51.15 & 60.00 & & & & \\
11 & 2.20 & 25.6 & 43.11 & & & & \\
\hline Total & $6.19^{\mathrm{a}}$ & $14.27^{\mathrm{a}}$ & $32.34^{\mathrm{b}}$ & Total & $9.77^{\mathrm{a}}$ & $39.81^{\mathrm{b}}$ & $65.59^{\mathrm{c}}$ \\
\hline
\end{tabular}

$a, b, c$ Superíndices diferentes entre valoraciones en el total indican diferencias significativas $(p<0.05)$

la presencia de ácidos grasos libres como consecuencia del efecto del tiempo sobre la oxidación de la grasa de la leche.

\section{Análisis Estadístico}

La evaluación del efecto antioxidante de la leche se realizó mediante un análisis de varianza teniendo en cuenta las variables tiempo de almacenamiento y tratamiento. La diferencia estadística entre tratamientos se determinó con una prueba de diferencia mínima significativa DMS.

Para el análisis de la calidad composicional de la leche se trabajó con los promedios de los parámetros evaluados y se aplicó la prueba «t» para pruebas independientes para determinar posibles diferencias.

\section{Resultados}

\section{Harina de Alfalfa}

La evaluación bromatológica de la harina de alfalfa comercial presentó $96.9 \%$ de materia seca, $8.7 \%$ de cenizas, $12.2 \%$ de proteína cruda, $41.1 \%$ de fibra cruda, $59.3 \%$ de fibra detergente neutra, $48.8 \%$ de fibra detergente ácida, $1.4 \%$ de grasa y $34.5 \%$ de extracto etéreo. Los animales tomaron entre 5 y 7 días en acostumbrarse a la ración.

\section{Efecto Antioxidante}

La concentración de ácido palmítico en la primera medición (Día 0) fue relativamente similar para el grupo tratado y el grupo 
Cuadro 2. Concentración de ácidos grasos (AG) en leche y grasa de leche de vacas suplementadas durante cuatro meses con harina de alfalfa y en vacas control

\begin{tabular}{|c|c|c|c|c|}
\hline \multirow[b]{2}{*}{ Ácido graso } & \multicolumn{2}{|c|}{ Grupo tratado } & \multicolumn{2}{|c|}{ Grupo control } \\
\hline & $\begin{array}{l}\text { g AG/100 } \\
\text { g de grasa }\end{array}$ & $\begin{array}{l}\text { g AG/100 } \\
\text { g de leche }\end{array}$ & $\begin{array}{l}\text { g AG/100 } \\
\text { g de grasa }\end{array}$ & $\begin{array}{l}\text { g AG/100 } \\
\text { g de leche }\end{array}$ \\
\hline Metil hexanoato & 1.00 & 0.03 & 0.92 & 0.03 \\
\hline Metil octanoato (metil caprilato) & 0.56 & 0.02 & 0.50 & 0.02 \\
\hline Metil decanoato (metil caprinato) & 1.31 & 0.05 & 1.00 & 0.04 \\
\hline Metil laurato & 1.55 & 0.06 & 1.45 & 0.05 \\
\hline Metil tridecanoato & -- & -- & 0.05 & 0 \\
\hline Metil tetradecanoato (metil miristato) & 7.71 & 0.31 & 7.36 & 0.30 \\
\hline Metil éster del ácido miristotélico & 1.66 & 0.06 & 1.77 & 0.07 \\
\hline Metil pentadecanoato & 1.41 & 0.05 & 1.61 & 0.06 \\
\hline Metil palmitato & 27.94 & 1.13 & 24.81 & 1.01 \\
\hline Metil palmitoleato & 1.20 & 0.04 & 1.30 & 0.05 \\
\hline Metil heptadecanoato & 0.88 & 0.03 & 2.17 & 0.08 \\
\hline $\begin{array}{l}\text { Metil éster de ácido cis-10- } \\
\text { heptadecanoido }\end{array}$ & 0.29 & 0.01 & 0.58 & 0.02 \\
\hline Metil octadecanoato (metil estearato) & 9.17 & 0.47 & 9.48 & 0.38 \\
\hline Metil éster del ácido trans-9-eladínico & 3.42 & 0.14 & 3.70 & 0.13 \\
\hline Metil éster del ácido cis-9-oleico & 23.40 & 0.94 & 27.33 & 1.13 \\
\hline Metil éster del ácido linolelaídico & 0.29 & 0.02 & 0.25 & 0.01 \\
\hline Metil linoleato & 2.02 & 0.08 & 1.57 & 0.06 \\
\hline Metil cis-11 eicosanotato & 1.54 & 0.06 & 0.24 & 0.01 \\
\hline Metil lignocerato & -- & -- & 0.42 & 0.01 \\
\hline
\end{tabular}

Cuadro 3. Calidad composicional de la leche de vacas de vacas suplementadas durante cuatro meses con harina de alfalfa $\mathrm{y}$ en vacas control

\begin{tabular}{lcc}
\hline & Tratadas & Control \\
\hline Grasa (\%) & 3.34 & 3.05 \\
Densidad & 24.82 & 25.9 \\
Sólidos totales (\%) & 12.2 & 12.75 \\
Proteína (\%) & 2.61 & 2.86 \\
Lactosa (\%) & 4.12 & 4.11 \\
pH & 6.4 & 6.5 \\
\hline
\end{tabular}

control (6.19 y $9.77 \mathrm{ng} / \mathrm{ml}$, respectivamente), en tanto que en la tercera medición (Día 14) las diferencias fueron marcadas $(32.34 \mathrm{y}$ $65.59 \mathrm{ng} / \mathrm{ml}$, respectivamente, $\mathrm{p}<0.05$ ) (Cuadro 1). La concentración de ácido palmítico aumentó con el tiempo en ambos grupos, mostrando el proceso oxidativo de la grasa; sin embargo, dicho proceso fue más rápido en los animales no tratados.

\section{Presencia de Ácidos Grasos}

De los AG evaluados, palmitato, metil linoleato, metil tetradecanoato, metil- 
decanoato, metil laurato, metil hexanoato, metil octanoato, metil éster del ácido linolelaídico y metil cis-11 eicosanotato tuvieron mayor concentración en grasa de la leche de los animales tratados $(\mathrm{p}<0.05)$. Asimismo, metil éster del ácido cis-9-oleico, metil heptadecanoato, metilpentadecanoato, metil palmitoleato, metilocatadecanoato, metil éster del ácido cis-9-oleico, metil éster del ácido trans-9-eladínico, metil éster de ácido Cis-10heptadecanoido y metil éster del ácido miristotélico se encontraron en menor concentración en la grasa de la leche de las vacas tratadas, teniendo una diferencia significativa $(\mathrm{p}<0.05)$ (Cuadro 2).

\section{Calidad Composicional de la Leche}

El porcentaje de grasa fue ligeramente superior en la leche de vacas suplementadas en comparación con la leche de las vacas control, aunque esta diferencia no fue significativa. Por otro lado, los porcentajes de proteína y sólidos totales y la densidad de la leche fueron ligeramente superiores en la leche de las vacas control, pero estas diferencias no fueron significativas, lo que sugiere que la suplementación con alfalfa no influyó en las características de la leche (Cuadro 3).

\section{Discusión}

La oxidación de la grasa de la leche fue menor en la leche de las vacas suplementadas, incluso a los 14 días de haberla obtenido y dejándola a temperatura ambiente. La capacidad de las isoflavonas de evitar o prevenir la oxidación de la grasa de los alimentos fue reportada por Moreno et al. (2007), quienes mediante pruebas in vitro (valor índice de peróxidos) demostraron la acción antioxidante de isoflavonas obtenidas de Citrus paradisi (pomelo, toronja) frente a aceites de soja.

En los grupos, tratado y control de este estudio, se pudo evidenciar que la concentra- ción de ácido palmítico, interpretada como indicador del proceso de oxidación de la grasa de la leche, fue similar el día cero, lo que significa que las muestras llegaron al laboratorio con un grado de oxidación bajo, por el poco tiempo que había transcurrido entre la toma y la valoración. Cuando la leche se mantuvo a temperatura ambiente, se observó que para los días 7 y 14, la grasa de la leche fue sufriendo proceso oxidativo, reflejado en el aumento progresivo de la concentración del ácido palmítico libre, que fue muy marcada para el grupo control en el día 14, día donde se encontró diferencia significativa con el grupo tratado. Comparando los resultados de la prueba en ambos grupos, se infiere que la suplementación con alfalfa generó un efecto antioxidante de la grasa de la leche, toda vez que la liberación de ácido palmítico fue menor a lo observado en el grupo control.

Los AG presentes en la leche, ante las condiciones de manipulación y transporte en temperaturas relativamente altas, propias de los países tropicales, son convertidos por procesos no enzimáticos en AG libres (AGL), que luego son transformados en AG saturados (AGS). Estos pueden sufrir enranciamiento u oxidación, procesos causantes de la descomposición no bacteriana de la leche (Páez et al., 2006). La oxidación de las cadenas de ácidos grasos de la grasa de la leche conduce a alteraciones químicas de sus compuestos y a sabores y olores desagradables; en el proceso se forman radicales libres que reaccionan con el oxígeno, formando hidroperóxidos que por ser inestables propagan la reacción oxidativa (MartínezMonteagudo et al, 2012). La oxidación de la grasa láctea requiere, como paso previo, la hidrólisis de los triglicéridos constituyentes y la liberación de los ácidos grasos, por acción de las lipasas, enzimas presentes y activas en leche, con el consiguiente descenso del $\mathrm{pH}$, lo que podría relacionar las variaciones del pH y el estrés oxidativo de la leche (Miranda et al., 2011). 
Las diferencias encontradas en $\mathrm{AG}$ en la leche de los dos grupos de vacas no generaron un cambio marcado en la composición de los AG, toda vez que ello depende de la dieta y fisiología propias de la especie. En este estudio se encontró mayor concentración de los AG saturados y menor proporción de los insaturados, en concentraciones que varían de un AG a otro, en concordancia con la fisiología digestiva y de la glándula mamaria. La leche bovina contiene $70-75 \%$ de AGS (laúrico, mirístico y palmítico), 20-25\% de AG insaturados $\mathrm{y}$ tan solo $5 \%$ de $\mathrm{AG}$ poliinsaturados, AGPI (linoleico), donde los primeros se asocian con problemas de hipercolesterolemia y alteraciones cardiacas en animales y humanos y los segundos son hipolipemiantes (Castro et al., 2014; Samková et al., 2014). En este estudio se encontraron diferencias entre unos y otros. Metil octanoato (metil caprilato) estuvo en concentración de $0.56 \%$ en vacas que recibieron alfalfa y $0.5 \%$ en aquellas que no la recibieron, valores por debajo de lo reportado por García et al. (2014) quienes indican un valor de $2.5 \%$. Situación similar se observa con el metil decanoato (metil caprinato) que el mismo autor reporta en concentración de $3.8 \%$ para la leche de vaca; sin embargo, la concentración del metil tetradecanoato (metil miristato) estuvo más alta en el presente trabajo. Por otro lado, el metilpalmitato mostró un comportamiento similar a lo que indica la literatura $(25 \%)$, ya que estuvo en concentración de $27.9 \%$ (suplementadas) y $24.81 \%$ (no suplementadas). Las diferencias encontradas se indica que corresponden a diferencias metabólicas ruminales asociadas con los diferentes tipos de dietas (García et al., 2014).

En ambos grupos de este estudio se pudo evidenciar que la concentración de ácido palmítico, interpretada como indicador del proceso de oxidación de la grasa de la leche, fue similar el día cero, lo que significa que las muestras llegaron al laboratorio con un grado de oxidación bajo por el poco tiempo que había transcurrido entre la toma y la valoración. Cuando la leche se mantuvo a temperatura ambiente, se observó que para los días 7 y 14 la grasa de la leche fue sufriendo proceso oxidativo, reflejado en el aumento en la concentración del ácido palmítico libre que fue muy marcada para el grupo control en el día 14 en el que se encontró diferencia estadísticamente significativa con el grupo tratado

La calidad de la leche de los animales de estudio estuvo dentro de los parámetros indicados para el país para leche cruda (MPS, 2006). No obstante, se esperaba un aumento en el porcentaje de proteínas, tal como lo reporta la literatura cuando se administra alfalfa (Doreau et al, 2014; Zyl et al., 2014). En forma similar, se esperaba un incremento en la grasa de la leche (Doreau et al., 2014; Samková et al., 2014; Zyl et al., 2014), así como aumento de los ácidos grasos monoinsaturados y disminución de los saturados (Doreau et al., 2014).

\section{Conclusiones}

- Las isoflavonas y sus metabolitos activos eliminados en la leche de vacas suplementadas con alfalfa pueden generar efecto antioxidante, capaz de retardar la oxidación de la grasa de la leche, prolongando su tiempo de vida útil.

- La adición de alfalfa en la ración diaria de vacas en producción influyó positivamente en la concentración de grasa de la leche, aumentando los AG poliinsaturados y disminuyendo los saturados.

- La suplementación con alfalfa no generó cambios marcados en la calidad composicional de la leche.

- La presencia de isoflavonas en la leche de vacas suplementadas con alfalfa y la modificación de los ácidos grasos presentes, puede significar al productor la obtención de un producto enriquecido, que fuera de los nutrientes propios de la leche, aporta al consumidor sustancias antioxidantes benéficas para la salud y ácidos grasos benéficos, sin afectar la salud del animal. 
Agradecimientos

Los autores del trabajo expresan su agradecimiento a la Dirección de Investigaciones de la Universidad Pedagógica y Tecnológica de Colombia por la financiación del proyecto y al personal de la Granja Experimental Tungüavita por facilitar la realización del trabajo.

\section{Literatura Citada}

1. Al-dosari M. 2012. In vitro and in vivo antioxidant activity of alfalfa (Medicago sativa L) on carbon tetrachloride intoxicated rats. Am J Chin Med 40: 779793. doi: 10.1142/S0192415X12500589

2. Antignac JP, Cariou R, Le Bizec B, Andre F. 2004. New data regarding phytoestrogens content in bovine milk. Food Chem 87: 275-281. doi: 10.1016/ j.foodchem.2003.12.013

3. Cartaya O, Reynaldo I. 2001. Flavonoides: características químicas y aplicaciones. Cultivos Trop 22(2): 5-14.

4. Castro H, González F, Domínguez A, Pinos J, Morales E, Vieyra R. 2014. Efecto del nivel de concentrado sobre el perfil de ácidos grasos de la leche de vacas Holstein en pastoreo. Agociencia. 48: 765-775.

5. De Feo V, Qaranta E, Fedel V, Claps $S$, Rubino R, Pizza C. 2006. Flavonoids and terpenoids in goat milk in relation to forage intake. Ital J Food Sci 18: 85-92.

6. Doreau M, Ferlay A, Rochette Y, Martin C. 2014. Effects of dehydrated lucerne and soya bean meal on milk production and composition, nutrient digestion, and methane and nitrogen losses in dairy cows receiving two different forages. Animal 8: 420-430. doi: 10.1017/S1751731113002206

7. García CAC, Montiel RLA, Borderas TF. 2014. Grasa y proteína de la leche de vaca: componentes, síntesis y modificación. Arch Zootec 63: 85-105.
8. Gohlke A, Ingelmann CJ, Nürnberg G, Starke A, Woffram S, Metges CC. 2013. Bioavailability of quercetin from its aglycone and its glucorhamnoside rutin in lactating dairy cows after intraduodenal administration. J Dairy Sci 96: 2303-2313. doi: 10.3168/jds.2012-6234

9. Hoikkala A, Mustonen E, Saastamoinen I, Jokela T, Taponen J, Saloniemi H; Wähälä K. 2007. High levels of equol in organic skimmed Finnish cow milk. Mol Nut Food Res 51: 782-786. doi: 10.1002/mnfr.200600222

10. Kilic M, Lindsay RC. 2005. Distribution of conjugates of alkylphenols in milk from different ruminant species. J Dairy Sci 88: 7-12. doi: $10.3168 / j d s . S 0022-$ 0302(05)72656-4

11. Leiber F, Kreuzer M, Nigg D, Wettstein HR, Scheeder MRL. 2005. A study on the causes for the elevated n-3 fatty acids in cows' milk of alpine origin. Lipids 40: 191-202. doi: 10.1007/ s11745-005-1375-3

12. Martínez-Monteagudo S, Saldaña M, Kennelly J. 2012. Kinetics of nonisothermal oxidation of anhydrous milk fat rich in conjugated linoleic acid using differential scanning calorimetry. J Therm Anal Calorim 107: 973-981. doi: 10.1007/s10973-011-1649-8

13. Miranda M, Gormaz M, Romero FJ, Silvestre, D. 2011. Estabilidad de la capacidad antioxidante y $\mathrm{pH}$ en leche humana refrigerada durante 72 horas: estudio longitudinal. Nutr Hosp 26: 722-728.

14. Morel I, Wyss U, Collomb M. 2006. Grünfutter- oder Silagezusammensetzung und Milchinhaltsstoffe. AgrarForschung 13: 228-233.

15. Moreno AMJ, Guaran CY, Belen CDR, Garcia-Pantaleon D, Medina MCA. 2007. Efecto de los extractos de flavonoides de harinas de cáscaras y semillas de pomelos sobre la estabilidad de aceite de soja. Grasas y Aceites 58: 351358.

16. [MPS] Ministerio de la Protección Social. 2006. Decreto número 616 del 28 de febrero de 2006 del Ministerio de 
Protección Social. Bogotá-Colombia. [Internet]. Disponible en http://www. ica.gov.co/getattachment/15425e0f$81 \mathrm{fb}-4111-\mathrm{b} 215-63$ e61 e9e9130/ 2006D616.aspx

17. Palladino RA, Buckley F, Prendiville R, Murphy JJ, Callan J, Kenny DA. 2010. A comparison between HolsteinFriesian and Jersey dairy cows and their F-1 hybrid on milk fatty acid composition under grazing conditions. J Dairy Sci 93: 2176-2184. doi: 10.3168/jds.2009-2453

18. Páez R, Pensel N, Sabbag N, Taverna M, Cuatrín A, Zalazar C. 2006. Changes in free fatty acid composition during storage of whole milk powder. Int J Dairy Technol 59: 236-241. doi: 10.1111/ j.1471-0307.2006.00270.x

19. Piotrowska, KK, Woc ${ }^{3}$ awek-Potocka II, Bah MM, Piskula MK, Pilawski W, Bober A, Skarzynski DJ. 2006. Phytoestrogens and their metabolites inhibit the sensitivity of the bovine corpus luteum to luteotropic factors. J Reprod Dev 52: 33-41.

20. Rana MG, Katbamna RB, Padhya AA, Dudhrejiya AD, Jivani NP, Sheth NR. 2010. In vitro antioxidant and free radical scavenging studies of alcoholic extract of Medicago sativa L. Rom J Biol 55(1): 15-22.

21. Samková E, Certíková J, Špièka J, Hanuš O, Pelikánová T, Kvác M. 2014. Eighteen-carbon fatty acids in milk fat of Czech Fleckvieh and Holstein cows following feeding with fresh lucerne (Medicago sativa L). Anim Sci Papers Reports 32: 209-218.

22. Setchell KDR, Faughnan MS, Avades T, Zimmer-Nechemias L, Wolfe BE, Brashear P, et al. 2003. Comparing the pharmacokinetics of daidzein and genistein with the use of 13C-labeled tracers in premenopausal women. Am J Clin Nutr 77: 411-419.

23. Shipe WF, Senyk GF, Fountain KB. 1980. Modified copper soap solvent- extraction method for measuring freee fatty acids in milk. J Dairy Sci 63: 193198. doi: 10.3168/jds.S0022-0302(80)82913-4

24. Steinshamn H, Purup S, Thuen E, Hansen-Moller J. 2008. Effects of clover-grass silages and concentrate supplementation on the content of phytoestrogens in dairy cow milk. J Dairy Sci 91: 2715-2725. doi: 10.3168/jds.20070857

25. Trinácty A, Krizova L, Schulzova V, Hajslova J, Hanus O. 2009. The effect of feeding soybean-derived phytoestogens on their concentration in plasma and milk of lactating dairy cows. Arch Anim Nutr 63: 219-229. doi: 10.1080/ 17450390902859739

26. Heather B, Patisaul HB, Dindo M, Whitten PL, Young LJ. 2001. Soy isoflavone supplements antagonize reproductive behavior and estrogen receptor $\alpha$ - and $\beta$-dependent gene expression in the brain. Endocrinology 142: 2946-2952. doi: 10.1210/ endo.142.7.8241

27. Woclawek-Potocka I, Mannelli C, Boruszewska D, Kowalczyk-Zieba I, Wavniewski T, Skarhynski DJ. 2013. Diverse effects of phytoestrogens on the reproductive performance: cow as a model. Int J Endocrinol ID 650984. doi: 10.1155/2013/650984

28. Woclawek-Potocka I, Bah MM, Korzekwa A, Piskula MK, Wiczkowski W, Depta A, Skarzynski DJ. 2005. Soybean-derived phytoestrogens regulate prostaglandin secretion in endome-trium during cattle estrous cycle and early pregnancy. Exp Biol Med 230: 189-199.

29. Zyl MV, Meesge R, Scholtz GDJ, Einkamerer OO. 2014. The effect of lucerne (Medicago sativa) hay quality on milk production and composition of Jersey cows. S Afr J Anim Sci 44: 25-30. 